\title{
Development of Online Computer Supported Collaborative e-Learning with Augmented Reality
}

\author{
Yu-Chuan Liu \\ Department of Information Management, Tainan University of Technology, Tainan 71002, Taiwan. \\ t00258@mail.tut.edu.tw
}

\begin{abstract}
This paper proposes an interactive augmented reality (AR) architecture for online computer supported collaborative work (CSCW) for e-Learning. Current CSCW applications for AR are focused on multiple users met together to collaborate on the same physical environment. The proposed architecture can provide collaboration through computer network without the need of face-toface communication. System architecture and operation scenario are firstly constructed. AR applications for e-Learning is studied. The proposed interactive AR system architecture can provide new experiences for users and both the effectiveness and efficiency for online training applications can be significantly improved.

Index Terms - Augmented Reality, Computer Supported Cooperative Work, e-Learning.
\end{abstract}

\section{Introduction}

Augmented Reality (AR) creates a mixed reality by augmenting the physical real-world environment with virtual computer-generated imagery. The information display and image overlay are context-sensitive [1]. AR has been known as a powerful tool to provide a brand-new perception to achieve the real world task, yields the need for more complicates and customized information interactions through computer-supported collaborative works. The AR modeler of Kiyokawa et al. [2] used a magnetic tracker to allow people to create AR contents. A annotation taxonomy system was proposed for online editing and creation of annotations with the interactivity dimension [3]. Dias et al. [4] applied 3D pointers that visual markers attached to rings or bracelets were used to recognize 3D gestures. Lu and Smith [5] proposed an e-commerce web page that integrated virtual product into an online shopper's physical environment for customers to realistically interact.

Entertainment applications of AR are becoming popular recently. Buchanan et al. [6] provided a game system that combining the physical input devices and AR system. An immersive system for multiplayer each wearing a HMD with head tracker for face-to-face collaboration was developed Tedjokusumo et al. [7]. Ellis et al. [8] proposed the computer supported cooperative work for a group of users to interact and cooperative on the shared objectives. Regenbrecht et al. [9] presented a novel vision-based AR system for multi-user collaborative application.

Current computer supported collaborative work (CSCW) environments with AR concentrated on multi-user interactions at the same workplace. The proposed architecture can provide collaboration through computer network without the need of face-to-face communication. Application scenario of AR for e-Learning is studied in this research. Applications of these
AR results will provide new experiences and effective tools for the CSCW.

\section{Computer Supported Collaborative Work with AR}

Virtual Reality (VR), focused on completely immersing the user inside a synthetic environment by creating virtual objects, has long been applied in fields of immersive entertainments and flight training simulators. On the contrary, AR allows the user to see the real world together with virtual objects superimposed upon or composited. Milgram and Kishino [10] described a spectrum that spans from entirely real environment to a purely virtual environment. In between are AR (closer to the real environment) and Augmented Virtuality (AV) (closer to the virtual environment) as shown Fig. 1. The real world pluses the virtual environment is called the Mixed Reality. AR lies near the real-world end of the spectrum with the predominate perception being the realworld augmented by computer generated data.

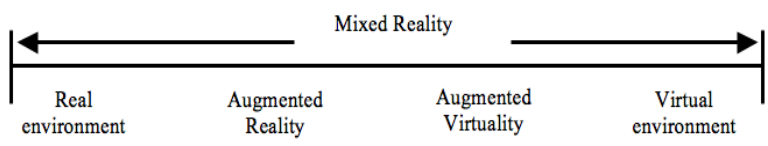

Fig. 1 Representation of a Reality-Virtuality spectrum [10].

A basic construction of AR environment needs hardware display, tracking markers, and computer vision techniques. Precise registration of real and virtual objects needs hardware display support. A camera integrated with computer to provide video capture capability is a more popular feature in $A R$ system because of the low cast and high flexibility. The tracking markers are composed of marked cards with square fiducial patterns. As the corresponding parameters of the 3D virtual model imported into the AR system and the markers are detected by camera, computer vision techniques will then be applied to identify the specific square marker to show the virtual objects. The $3 \mathrm{D}$ virtual model will be appeared precisely registered with the calculated markers positions. The workflow can be summarized in Fig. 2. Software platform plays an important role of connecting segment for markerbased tracking of computer vision in AR system. Vizard can provided high quality, low-cost immersive $3 \mathrm{D}$ products, and supported the ARToolKit library. The programming language used in Vizard is called Python, an intuitive language similar to $\mathrm{C}++$ or Java. The open-source language Python is free and will be applied for software platform. 
In face-to-face collaboration, people use speech, gesture, gaze, and nonverbal cues for communication. AR system had also been applied and identified as an important means of CSCW. Szalavari [11] summarized five key attributes of collaborative AR environments as: (1) objects that don't exist in the real world can be viewed and examined; (2) real objects can be augmented with virtual annotations; (3) multi-user can see each other and cooperate in natural ways; (4) individual users control their own independent viewpoints; (5) displayed data can be different for viewers depending on their personal needs and interests. Ohshima et al. [12] developed the AR2 Hockey interface for two users to play air hockey by using a virtual puck. Regenbrecht et al. [9] proposed CSCW environments to facilitate multi-user in a face-to-face collaborative on the same job. In their displays, they can see video of the real world with graphics superimposed.

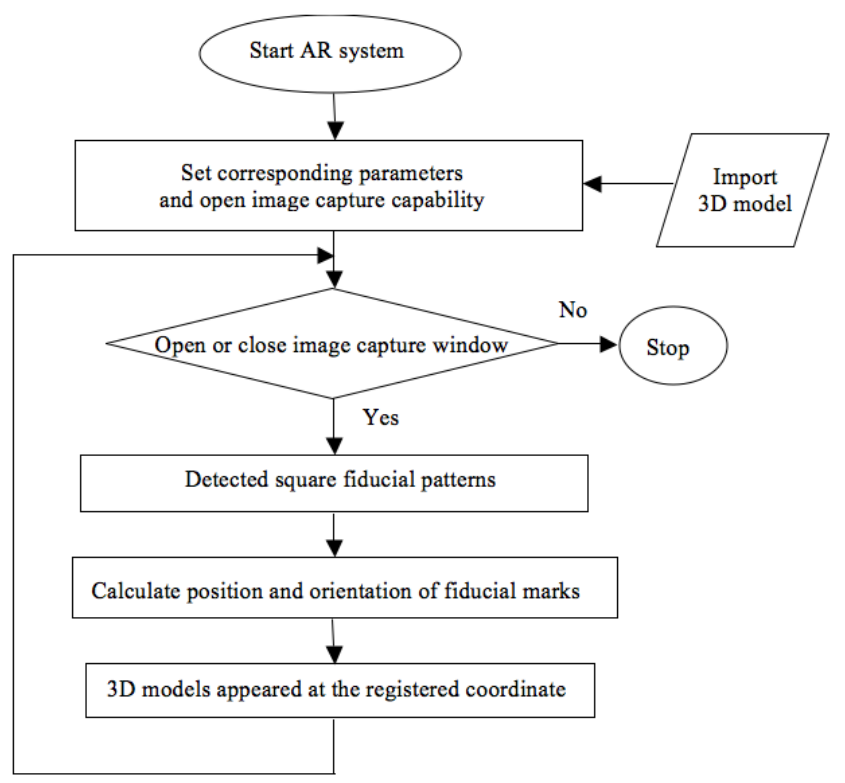

Fig. 2 Workflow of the AR system.

\section{Online Collaboration for e-Learning with AR}

The network structure for the demonstrative example is shown in Fig. 3. The collaborative tangible AR environment is conducted by the architecture shown in Fig. 4. Consider two users, $\mathrm{A}$ and $\mathrm{B}$, the $\mathrm{AR}$ systems are started respectively in different computers connected by network. In order to perform the AR interaction, these two computers need to be identified by locating the IP address. Users A and B have their own web camera, display, and tracking markers. For the case that user A triggers an interactive event, the results of this triggered event will be shown simultaneously in the display monitors of both users $\mathrm{A}$ and $\mathrm{B}$. The event user $\mathrm{A}$ triggered will also be sent to the computer of user B and acted as a notice for user B to begin his trigger event. Multicast communication allows a single message send to a group of receivers. In order to increase interactivity, the real-time string communication between the remote computers by cPickle library with Python is applied to send commands and data transmission. Researches addressing on audio medium with AR are rare. ARGroove [13] allowed the user to remix and modulate musical elements and serve as musical containers by grouping related elements of musical composition together. This paper proposed another way to combine AR with sound effect for

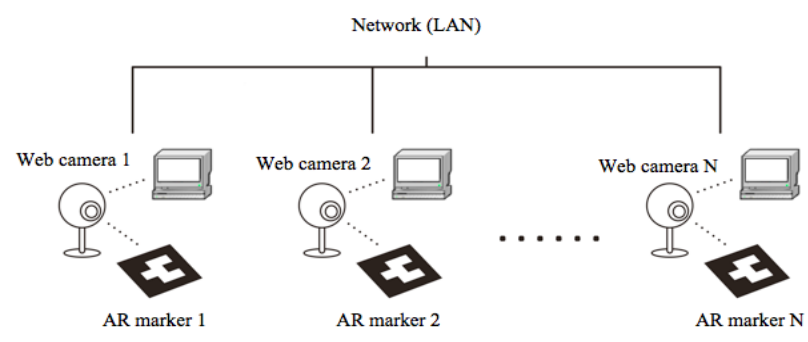

CSCW.

(a)

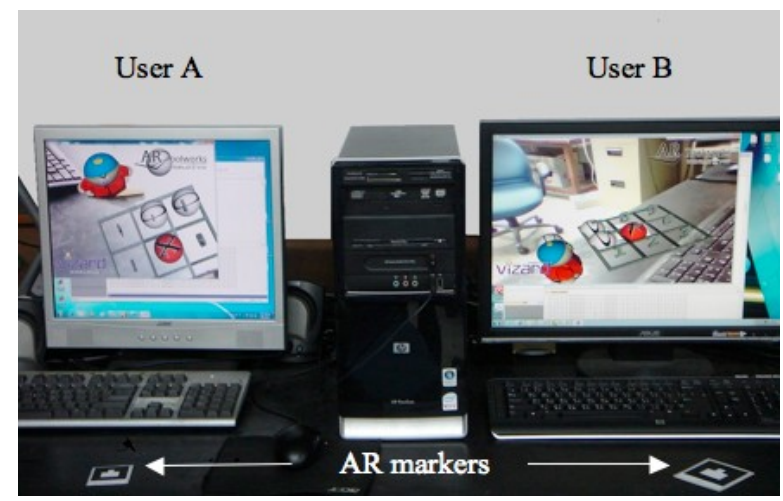

(b)

Fig. 3 Network structure of this paper: (a) schematic diagram and (b) system layout

The scenario of piano four hands online practicing CSCW environments is developed as an illustration. Piano four hands is a specific form of duet for a single piano with two players, often need to lay out with high and low pitch with the players playing separate segment to implement the full composition. To simplify the musical part of this work, this paper only uses just a few chords to create a basic song. As user A presses the keyboard button, the sound effect will be triggered, and the virtual keyboard button will be pressed simultaneously shown in the other user's monitor as shown in Fig. 5. User A also can receive the sound effect created from user B. User A and B can interact with each other to build a complete melody. The interactive AR system can also be applied in online piano teaching. Instead of face-to-face teaching, tutor can teach via network and interact with students to provide the same service. The proposed system also supporting media player interface that player can use guide marker to show the coaching video. By covering the marker, video can be controlled to play or stop as shown in Fig. 6. The proposed system is expected to be an attractive tool for students to overcome the location and distance limitations. 


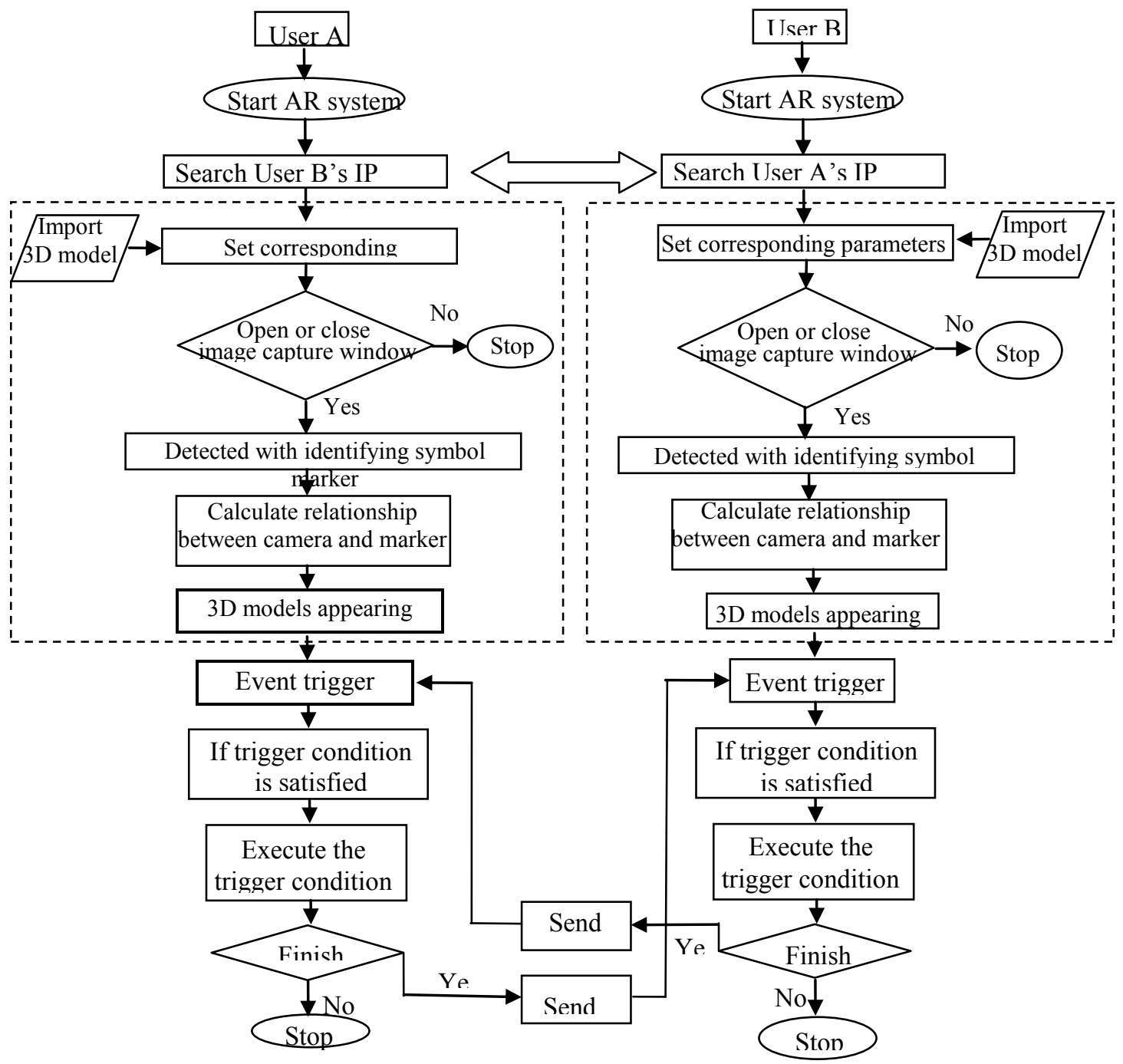

Fig. 4 Workflow of the proposed online AR CSCW.

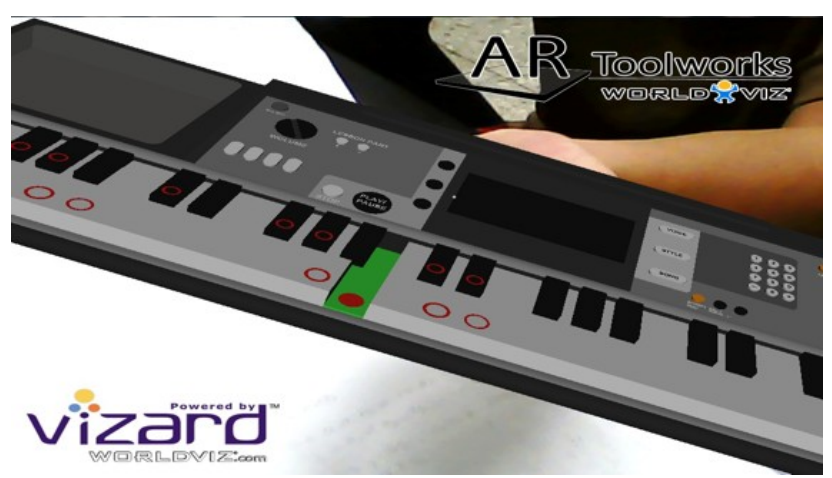

(a)

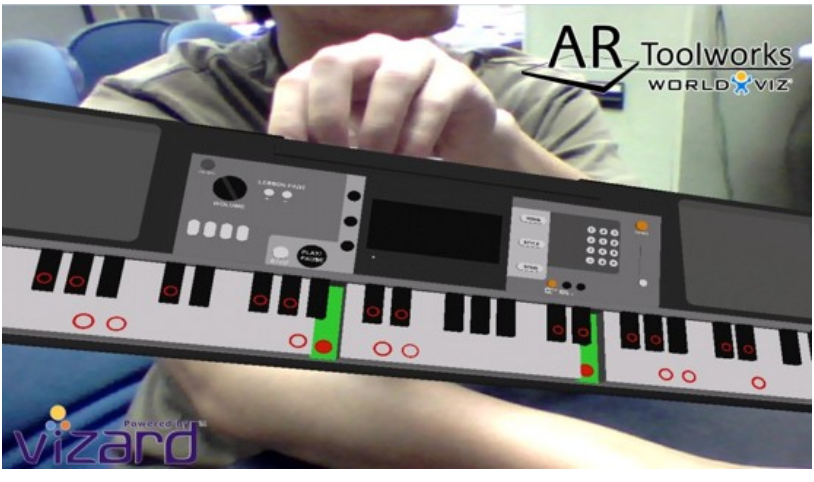

(b)

Fig. 5 Interactive AR system with audio effect for piano four hands, (a) piano played by user A, (b) piano played by user B. 


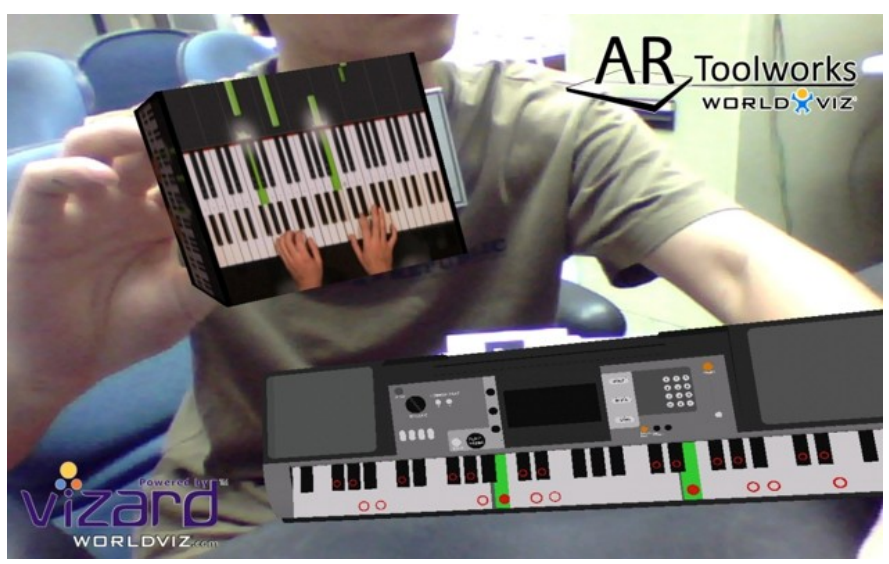

Fig. 6 Coaching video activated by tutor to provide instructions for student.

\section{Conclusion}

The interactive AR systems are constructed for CSCW in this paper. AR system architecture and application scenarios for multi-user online e-Learning in computer network are developed. AR can be applied as a technology to perform collaboration without the need of face-to-face communication. This collaborative AR system can provide a very real working simulation for users in different locations to practice their works. The proposed interactive AR system can provide new experiences for application field. Both the effectiveness and efficiency for online e-Learning application can be significantly improved.

\section{References}

[1] R.T. Azuma, Y. Baillot, R. Behringer, S. Feiner, S. Julier, and B. MacIntyre: IEEE Comp. Graph. and App., Vol.21 (2001, 6), pp. 34-47.

[2] K. Kiyokawa, H. Takemura, and N. Yokoya: IEEE Int. Conf. on Systems Man and Cybernetics, Tokyo, Japan (1999), pp. 48-53,

[3] J. Wither, S. DiVerdi, and T. Hollerer: Comp. \& Graph., Vol. 33 (2009, 6), pp. 679-689.

[4] J.M.S. Dias, P. Santos, and P. Nande: 2nd IEEE Int. Augmented Reality Toolkit Workshop, Tokyo, Japan (2003), pp. 29-31.

[5] Y. Lu and S. Smith: J. of Comp. and Info. Sci. in Eng., Vol.10 (2010, 2), pp. 021005.

[6] P. Buchanan, H. Seichter, M. Billinghurst, and R. Grasset: Int. Conf. on Adv. in Comp. Entertain. Tech., New Zealand (2008), pp. 17-20.

[7] J. Tedjokusumo, S.Z. Zhou, and S. Winkler: IEEE Trans. on Sys., Man, and Cyber., Vol.40 (2010, 1), pp. 147-157.

[8] C. A. Ellis, S.J. Gibbs, and G. Rein: Comm. of ACM, Vol. 34 (1994, 1), pp. 39-58.

[9] H.T. Regenbrecht, M. Wagner, and G. Baratoff: Virt. Real., Vol. 6 (2002, 3), pp. 151-166.

[10] P. Milgram and F. Kishino: IEICE Trans. on Info. Sys., Vol. E77-D, (1994, 12), pp. 1-15.

[11] Z. Szalavári, D. Schmalstieg, A. Fuhrmann, and M. Gervautz: Comp. Sci. Virt. Real., Vol.3 (1998, 1), pp. 37-48.

[12] T. Ohshima, K. Satoh, H. Yamamoto, and H. Tamura: IEEE Virt. Real. Ann. Int. Symp., Vol.1 (1998, 18), pp. 268-275.

[13] I. Poupyrev, R. Berryr, and J. Kurumisaea, "Augmented Groove: Collaborative Jamming in Augmented Reality," SIGGRAPH 2000 Conference Abstracts and Applications, ACM Press, pp.77, New York, 2000. 